\title{
ENDOSCOPIC REPAIR OF CSF RHINORHEA
}

*Mohamed Elsayed, **Ibrahim Abd-Elhady, ***Ahmed Abd-Elbaset

\begin{abstract}
Cerebrospinal fluid (CSF) rhinorrhea was first described by Galen, 200 B.C. Many attempts to correct a SF leak were done in the $20^{\text {th }}$ Century, although the first well-succeeded surgical approach was attributed to Dandy in 1926, when he sutured the fascia lata over dural defect, on back of the posterior wall of the frontal sinus, by intracranial route. In1981, when Wigand repaired fistulas using an endoscope, this approach became widely adopted. Fluid leakage may be expressed by several symptoms and/or signs, although rhinorrhea is the most frequent one. In addition, presence of SF may be a risky condition for patient and onset of infections in the central nervous system, such as meningitis. Thus, in the absence of spontaneous resolution, a corrective procedure should be performed. Today, with improvements of skull base surgeries and the introduction of functional endoscopic surgery of paranasal sinuses in the otorhinolaryngology's routine, there has been increased incidence of severe complications, such as the cerebrospinal fluid leakage 5. Thus, endonasal duraplasty has been successfully used to repair several dura mater lesions with low patient's morbidity rate. Between 2001 and 2006, 12 patients with cerebrosp-inal rhinorrhea were operated on using intranasal endoscopic approach. The charts of all patients treated in our hospital were reviewed. Twelve patients, 7 women $(58 \%)$ and 5 male $(42 \%)$, were included in the study. Patients' ages ranged from 18 to 58 years (mean: 39.3 years). Etiology, site of leakage, diagnosis, technique, cause of failure and follow-up are discussed. The authors concluded that transnasal endoscopic surgery for CSF rhinorrhea had high success rate, low morbidity and stable long-term results.
\end{abstract}

\section{MATERIAL AND METHODS:}

Between 2000 and 2006, 12 patients diagnosed with cerebrospinal fluid rhinorrhea were submitted to endonasal endoscopic surgery. All patients' medical files were revised in our service. Twelve patients $-7(58 \%)$ women and 5 male (42\%) - were evaluated in the study. Age varied from 18 to 58 years (mean: 39.3). Information related to cause, site of dural lesion, repairing technique, complications and follow-up were collected from the files. All patients underwent computed tomography (CT) (Fig. 1, 2, 3) eight underwent magnetic resonance imaging (MRI), and one underwent CT-cisternography.

Only MRI localized sites of leakage. T2-weighted MRI showed an arachnoid pouch prolapsing through the basal defect in two patients and hyperintense CSF leakage into the sinus in four patients or into the nasal cavity in one. CT and CT-cisternography showed fractured sites in patients with post-traumatic rhinorrhea but were inconclusive regarding the exact location of the site of CSF.

"Onlay" and "Underlay" techniques were used for correction of dural defect. All fistulas were repaired with connective tissues, such as free-mucosa flaps, Liofilized dura, septal bone and cartilage, perichondrium or fascia lata and were supported with gel foam and Surgical. In order to check success or failure rate during procedure, clinical exams, endoscopic controls were performed at late postoperative phase.

* ENT depart. Ahmed Maher Teaching Hospital

** ENT depart. Banha Teaching Hospital.

*** ENT depart. AL Azhar University.

\section{RESULTS:}

Causes of cerebrospinal fluid fistula and the sites in our series are listed in Table 1.

The most frequent site of fistulas was the ethmoidal cribriform area. Meningoencephalocele was the most prevalent cause of fistula. "Underlay" technique was used in 10 cases $(83 \%)$ (Fig. 4, 5, 6), while "onlay" was performed in 2 cases $(17 \%)$.

\begin{tabular}{|l|c|}
\hline Cause of CSF & No. of Cases \\
\hline Post-operative & 2 \\
\hline Trauma & 3 \\
\hline Meningoencephalocele & 4 \\
\hline Spontaneous & 3 \\
\hline TOTAL & 12 \\
\hline
\end{tabular}

Table 1. Cause of SF leakage site in patients treated by endonasal technique $(\mathrm{n}=12)$

"Onlay" technique was used in the patients who were also undergoing "underlay" technique (Fig. 7). Surgical and fat was used to fill the sphenoid sinus when SF was detected. Liofilized dura was used in 2 cases, while mucous flap was used in 8 cases. Fascia lata was used in 4 cases. External lumbar shunt was performed in one patient. Patients remained in relative rest and in decubitus with maximal inclination of $10^{\circ}$ for 5 days, on average, and lumbar shunt was maintained for 4 days. Laxative diet and instructions to avoid Valsalva were recommended to patients. Nasal packing was not used and prophylactic antibiotic was introduced during anesthesia induction. 
Mohamed Elsayed, et al

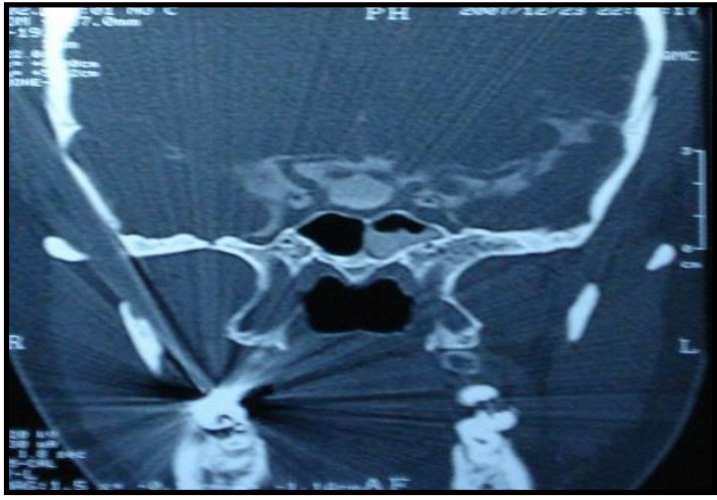

Fig. 1: CT of CSF of it sphenoid sinus

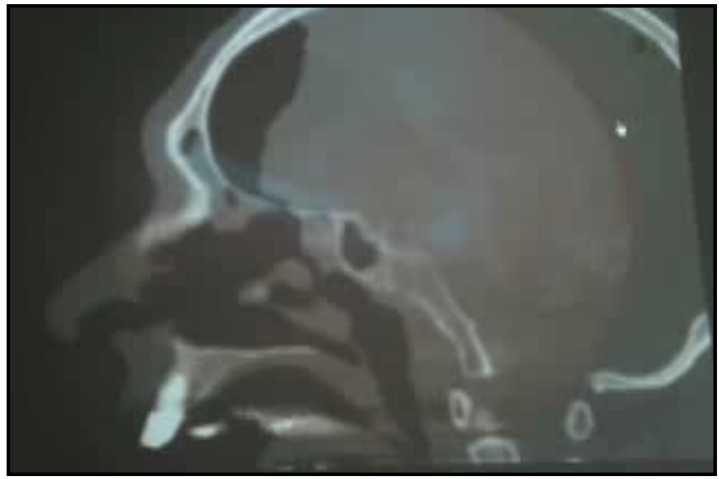

Fig. 2: Pre -operative CT of CSF of ethmoid sinus

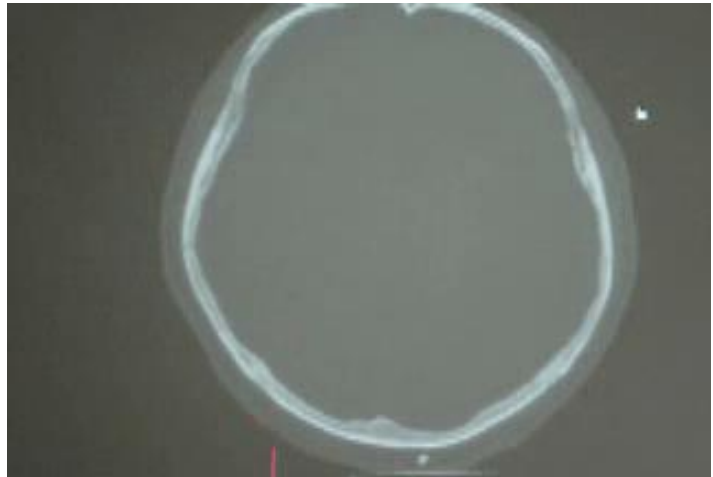

Fig. 3: Post -operative CT of CSF of ethmoid sinus

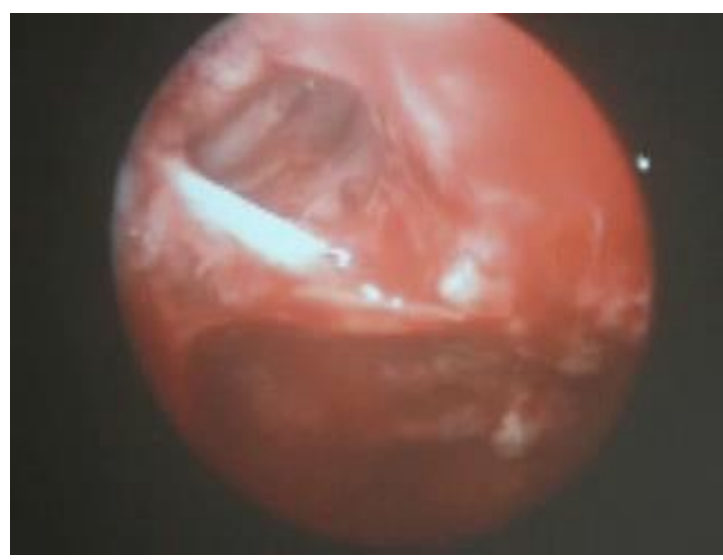

Fig. 4: Operative view of the defect in roof of ethmoid sinus

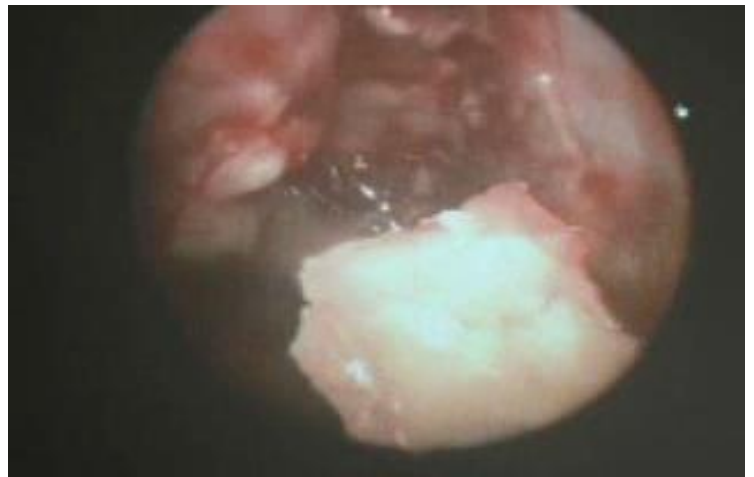

Fig. 5: Septal bone used in closing the defect in ethmoid sinus

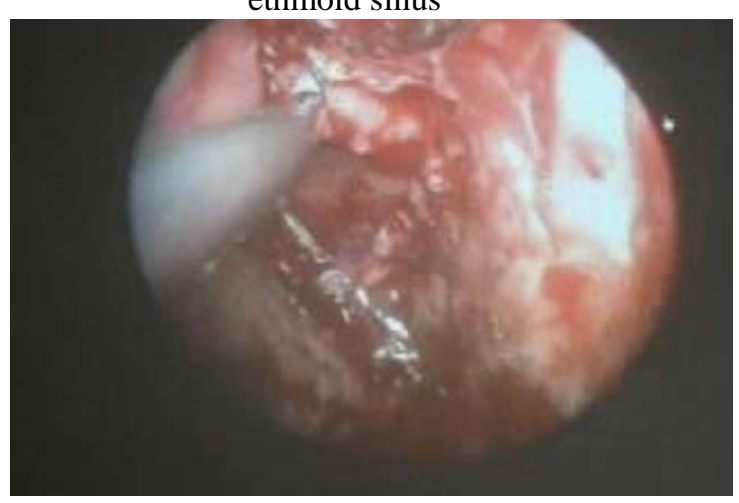

Fig. 6: Underlay bone graft.

We preferred using ceftriaxone, third generation cephalosporin with penetration in the hematoencephalic barrier. Postoperative follow-up varied from 5 to 50 months (mean: 36.4 months). One case presenting extensive bone failure was submitted to craniotomy for fistula repair by the neurosurgery service. Only one patient developed postoperative meningitis, although with complete recovery after antibiotic therapy.

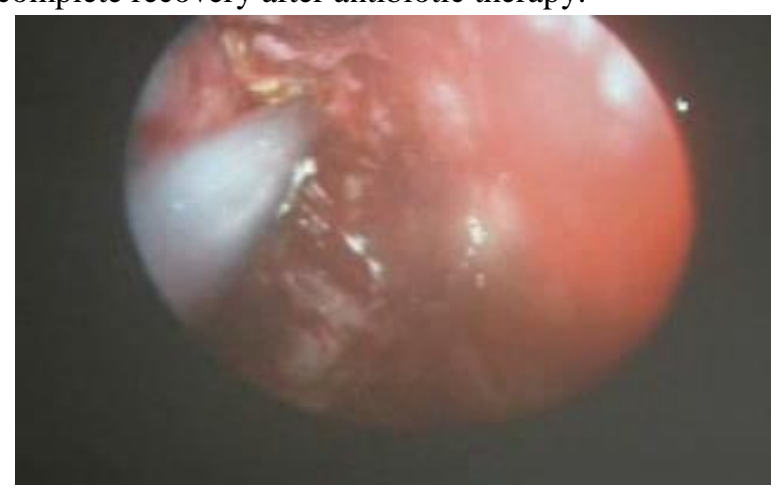

Fig. 7: Overlay fascia lata

\section{DISCUSSION:}

Communication between the subarachnoid space and the nasal cavity is called cerebrospinal fluid rhinorrhea (CSF) $(1,8)$ which can occur directly from the anterior cranial fossa and the nasal cavity, or indirectly from the middle and posterior fossa through the auditory tube. ${ }^{(1)}$ CSF's may be classified into traumatic and non-traumatic. ${ }^{(1,9)}$ In our series, $5(41.6 \%)$ of (CSF) were caused by trauma (2 


\section{Endoscopic Repair....}

surgical traumas and 3 non-surgical traumas), and the most affected site was the cribriform lamina and the posterior ethmoidal region, where bone is thinner and dura's adherence to this bone is stronger. ${ }^{(1)}$ Presence of spontaneous SF is variable in the literature, ranging from 4 to $39 \%$ of all cases of (CSF). Recently, this prevalence has increased to between 14 and $46 \%$ \%. $(12,13)$ The reason for this increase in the recent literature is unclear, although better recognition of this clinical entity and recent improvement in the quality of diagnostic tools may contribute to it. Our findings were similar to those reported by Wax et al. (1997), in which spontaneous leakages represented $25 \%$ in our cases. Origin of spontaneous leakage remains unclear, however some studies presented explanations such as: a congenital disorder; representation of a small meningocele eroding through bone or deriving from a focal atrophy of olfactory nerve filaments in the cribriform lamina. ${ }^{(1,6)}$ During preoperative evaluation and in order to locate the lesions site, all patients were submitted to computed tomography, with axial and coronal sections sinuses. We have not used B2transferrin, which is the most sensitive test to confirm SF leakage ${ }^{(5)}$ since it was not available, while magnetic resonance (MRI) was requested only for suspected cases of meningoencephaloceles, differently from Nachtigal and Schick studies, which proposed MRI for all cases. $(2,4)$ Fluorescein is still in use but is not preferred because it is associated with complications like transverse myelitis and allergic reactions. ${ }^{(14)}$ These complications were mainly related to errors in dosages, and the lack of premedication. Recent reports recommended premedication with dexamethasone and diphenhydramine to significantly reducing the risk of complications. ${ }^{(15,16) .} \mathrm{CSF}$ correction by endonasal technique has been broadly performed and the world literature shows that it is a secure and efficient method, has lower morbidity rates, higher success rates, and seldom results in anosmia. (17-20) They provide the best exposure of the sphenoid, parasellar, and posterior ethmoidal regions and offer excellent visualization of fistulas in the posterior wall of the frontal sinus, the cribriform plate, and the fovea ethmoidalis. ${ }^{(17,19-22)}$ Transnasal endoscopic surgery minimizes intranasal trauma and preserves the bony framework supporting the frontal recess and other critical areas. (25) In contrast, exposure of the skull base and the necessity of brain retraction during intracranial procedures are associated with a significant risk of anosmia, postoperative intracerebral hemorrhage, and brain edema. ${ }^{(17)}$ The failure rate associated with the management of CSF leaks via an intracranial approach has ranged from 20 to $40 \%$. ${ }^{(18,26)}$ .Localization and visualization by endoscope allow the surgeon to more easily remove the fibrous tissue and scarify the lesion's borders, which yield greater graft adherence in the region with excellent outcomes. $(1,10)$ There are several distinct endonasal techniques for SF correction and all have been successful. ${ }^{(2,5)}$ In addition, a variety of autologous and heterologous grafts derive from connective tissues. In our sample, as well as in the literature mentioned, we have not observed surgical failures associated with a specific type of graft used.
According to Schick (2001) and Hao (1996), incomplete exposure and large bone defect were prevalent factors in therapeutic failures. ${ }^{(2,10)}$ In our series, success rate of first intervention was of approximately $92 \%$, which is comparable to results previously reported in the literature. (12) Failures were probably due to large defects, such as hernia of meningoencephalic sac at the cribriform area or in the sphenoid sinus or to extensive front-basal fractures. Non-recognition of dural defect adjacent to primary defect was the cause of failure in the series of Hao et al., ${ }^{(10)}$ in these cases, due to high deficit of fistulas, there was impairment in graft placement and insufficient adherence. We observed that postoperative fistulas from endoscopic surgery affected the cribriform lamina and posterior ethmoid, a fragile region in the skull base to which attention should be given in suspected cases of CSF. ${ }^{(4,10)}$ Meningitis is the most feared life-risk complication in patients with CSF. ${ }^{(11)}$ Studies demonstrate incidence in up to $40 \%$ of patients. We only had one case of meningitis associated with large bone defect, which corroborates low morbidity of this endonasal technique. Postoperative patients should be carefully assisted. Use of prophylactic antibiotics is controversial. (11) We used antibiotics in all patients; however, Nachtigal (4) who had not adopted a prophylactic approach had no cases of meningitis in his 12-patient series. Among 115 patients with accidental CS leakage, Choi (11) observed a higher incidence of meningitis in patients who underwent antibiotic prophylaxis.

\section{CONCLUSION:}

Endonasal fistula repair is the approach of choice to reach high success rate and low morbidity in the treatment of cerebrospinal fluid rhinorrhea. CSF leakage associated with large defects, meningoceles and secondary lesions should receive special attention, while many times technique modifications are necessary to assure continuous fistula closure.

\section{REFERENCES:}

1. Wax MK, Ramadan HH, Ortiz O, Wetmore SJ. Contemporary management of cerebrospinal fluid rhinorrhea. Otolaryngology- Head and Neck Surgery 1997; 116 (4): 442-9.

2. Schick B, Ibing R, Brors D, Draf W. Long-term study of endonasal duraplasty and review of the literature. Ann Otol Rhinol Laryngol 2001; 110: 142 7.

3. Vrabec DP, Hallberg OE. Cerebrospinal fluid rhinorrhoea by endonasal surgery. Arch Otolaryngol 1964; 80: 218-29.

4. Nachtigal D, Frenkiel S, Mohr G. Endoscopic repair of cerebrospinal fluid rhinorrhea: Is it the treatment of choice. The journal of Otolaryngology 1999; 28 (3): 129-33. 


\section{Mohamed Elsayed, et al}

5. Jones ME, Reino T, Gnoy A et al. Identification of intranasal cerebrospinal leaks by topic application with fluorescein dye. American journal of rhinology 2000; 14 (2): 93-6.

6. Ramsden JD, Corbridge R, Bates G. Bilateral cerebrospinal fluid rhinorrhoea 2000; 114: $137-8$.

7. Burns JA, Dodson EE, Gross CW. Transnasal endoscopic repair of cranionasal fistulae: a refined technique with long-term followup. Laryngoscope 1996; 106: 1080-3.

8. Reyt E, Righini C, Colombani JM, Favre JJ. Transnasal endoscopic identification and repair of cerebrospinal rhinorrhoea. Skull Base Surgery 1997; 7 (Suppl 2): 52.

9. Ommaya AK. Spinal fluid fistulae. Clinics of Otolaryngology 1983; 8: 317-27.

10. Hao SP. Transnasal endoscopic repair of cerebrospinal fluid rhinorrhoea: an interposition technique. Laryngoscope 1996; 106: 501-3.

11. Choi D, Span R. Traumatic cerebralspinal fluid leakage: risks factors and the use of prophylactic antibiotics. Br J of Neurosurgery 1996; 10:571-5.

12. Dunn CJ, Alaani A, Johnson AP. Study On spontaneous cerebrospinal fluid rhinorrhoea: its aetiology and management. J Laryngol Otol. 2005; 119(1):12-15

13. Mirza S, Thaper A, Mc Clelland L, Jones NS. Sinonasal cerebrospinal fluid leaks management of 97 patients over 10 years. Laryngoscope. 2005; 115(10):1774-1777.

14. Mahaley MS, Odom GL. Complications following intracranial injections of fluorescein. J Neurosurg. 1966; 25:298-299.

15. Placantonakis DG, Tabaee A, Anand VK, Hiltzik D, Schwartz TH. Safety of low-dose intrathecal fluorescein in endoscopic cranial base surgery. Neurosurgery. 2007;61(3suppl):161-5.
16. Schlosser RJ, Bolger W. Endoscopic management of CSF rhinorrhoea. Otolaryngol Clinic North Am. 2006; 39(3):523-38.

17. Calcaterra TC. Extracranial surgical repair of cerebrospinal fluid rhinorrhoea. Ann Otol Rhinol Laryngol. 1980; 89:108-116.

18. Park JI, Strelzow VV, and Friedman WH. Current management of cerebrospinal fluid rhinorrhoea. Laryngoscope. 1983; 93:12941300REFERENCES.

19. Mattox DE, Kennedy DW. Endoscopic management of cerebrospinal fluid leaks and cephaloceles. Laryngoscope. 1990; 100:857-862.

20. Dodson EE, Gross CW, Swerdloff JL, Gustafson LM. Transnasal endoscopic repair of cerebrospinal fluid rhinorrhoea and skull base defect: a review of twenty-nine cases. Otolaryngol Head Neck Surg. 1994; 111:600-605.

21. McCormack B, Cooper PR, Persky M, Rothstein S. Extracranial repair of cerebrospinal fluid fistulas: technique and results in 37 patients. Neurosurgery. 1990; 27:412-417.

22. Briant TDR, Snell E. Diagnosis of cerebrospinal rhinorrhoea and the rhinologic approach to its repair. Laryngoscope. 1976; 77:1390-1409.

23. McCabe BF. The osteomucoperiosteal flap in repair of cerebrospinal fluid rhinorrhoea. Laryngoscope. 1976; 86:537-539.

24. Montgomery WW. Surgery of cerebrospinal fluid rhinorrhoea and otorrhoea. Arch Otolaryngol. 1966; 84:92-104.

25. Schaefer SD, Manning S, Close LG. Endoscopic paranasal sinus surgery: indications and considerations. Laryngoscope. 1989; 99:1-5. 